International Journal of Electrical and Computer Engineering (IJECE)

Vol. 9, No. 3, June 2019, pp. 1620 1629

ISSN: 2088-8708, DOI: 10.11591/ijece.v9i3.pp1620-1629

\title{
Crosstalk in misaligned free space optical interconnects: modelling and simulation
}

\author{
Nedal Al-Ababneh \\ Department of Electrical Engineering, Jordan University of Science \& Technology, Jordan
}

\begin{tabular}{l} 
Article Info \\
\hline Article history: \\
Received Jun 11, 2018 \\
Revised Dec 2, 2018 \\
Accepted Dec 27, 2018 \\
\hline Keywords: \\
Collins diffraction integral \\
Laguerre-Gaussian (LG) beam \\
Complex Gaussian functions \\
Optical crosstalk \\
Optical interconnects
\end{tabular}

Article Info

Article history:

Received Jun 11, 2018

Revised Dec 2, 2018

Keywords

Collins diffraction integra

Complex Gaussian functions

Optical interconnects

\begin{abstract}
We introduce convenient model and an optimization scheme to optimize the signal-to-crosstalk ratio (SCR) in a free space optical interconnects (FSOIs) system that uses microlenses with finite circular apertures. In this model, we consider both the stray light crosstalk and the crosstalk due to the diffraction at the microlens apertures to evaluate the SCR. Using cylindrical form of Collins diffraction integral and the Laguerre-Gaussian (LG) beam model, we derive an approximate closed form formula for the optical field of a multimode LG beam propagating through circular apertured FSOIs by expanding the hard edge circular aperture function of the microlens in terms of complex Gaussian functions. The analyses indicate that the size of the detector is an important factor to optimize the SCR for both the apertured and the unapertured misaligned FSOIs system. The effect of higher order mode of the laser source on the SCR is also considered.
\end{abstract}

\section{Corresponding Author:}

Nedal Al-Ababneh,

Department of Electrical Engineering,

Jordan University of Science \& Technology, Irbid, Jordan.

Email: nedalk@just.edu.jo

\section{INTRODUCTION}

The need for high density and high speed interconnects is demanding for high speed digital applications. Free space optical interconnects (FSOIs) can be used as data links with high aggregate bandwidth in digital systems [1]-[6]. These optical interconnects systems can be implemented using vertical cavity surface emitting lasers (VCSELs) array at the transmitter side and photodetectors array at the receiving side connected together by an optical free space channel containing microlens array to collimate the light and limit the light spreading.

Despite using microlens arrays, crosstalk between neighboring optical channels, due to stray light and the light diffracted from hard apertures of microlens, is still one of the major limiting factors that determine the performance of the FSOIs including the signal-to-crosstalk ratio (SCR), channel density, the interconnects capacity [4]-[6].Therefore, we consider it one of the major issues to study the impact of crosstalk on FSOIs systems and develop methods and models to describe the propagation of the light in these systems. Geometrical ray tracing methods can be used to describe the propagation of light beams in unapertured optical systems [6] and do not take into consideration the diffraction and the clipping of the light due to finite apertures. In reality, optical systems have limited size and in turn finite aperture. Therefore, methods to deal with these apertured systems are needed. In [1] a diffraction formalism based on HuygensFresnel principle was used to study the crosstalk effect in FSOIs taking into account the microlens finite aperture. The optical wave emitted from the VCSEL was a plane wave diffracted by a finite aperture window. In [7] a modified mode expansion was used to study the diffraction of the laser beam. In [5] ray tracing and diffraction propagation methods was combined and used to propagate the light in the optical interconnect system. However, these methods can be used to deal with aligned FSOIs systems. In practical 
applications, the optical components are subject to misalignment when constructing the FSOIs systems [8, 9]. In fact, misalignment of optical components degrades the performance of FSOIs, increases the crosstalk, and decreases system efficiency. Therefore, it is important to study the crosstalk induced by the misalignment of FSOIs. In [10], a method combining free-space Gaussian beam propagation theory and generalized Collins diffraction theory was presented to propagate the beams in a misaligned FSOIs system with finite aperture. The analysis in [10] assumed the fundamental Hermite-Gaussian model $\left(\mathrm{HG}_{00}\right)$ for the light beams in rectangular coordinate system.

Solutions of the paraxial Helmholtz equation can be found as combinations of Hermite-Gaussian modes (whose amplitude profiles are separable in $\mathrm{x}$ and $\mathrm{y}$ using Cartesian coordinates) or similarly as combinations of Laguerre-Gaussian modes (whose amplitude profiles are separable in $r$ and $\theta$ usingcylindrical coordinates). Although there are other possible modal decompositions, these two solutions are the most useful for problems with compact beams in which the optical power is confined along the optical axis. Beam profiles which are circularly symmetric (or lasers with cavities that are cylindrically symmetric) are often best solved using the Laguerre-Gaussian modal decomposition. These beams are defined in cylindrical coordinates and can have advantages over Hermite-Gauss modes in the presence of cylindrical symmetry.

In this paper, a crosstalk model for a misaligned lens based FSOIs system with circular aperture will be presented assuming Laguerre-Gaussian model of any order $\left(\mathrm{LG}_{\mathrm{pm}}\right)$ for the beam in a cylindrical coordinate system. This model is derived based on the cylindrical form of Collins diffraction integral. An approximate analytical formula for the optical field at the receiver side is derived by expanding the microlens aperture function in terms of complex Gaussian functions. Compared to other models, this model is more appropriate to deal with the circular symmetric optical elements of the FSOIs which correspond to the highest possible symmetry and thus high interconnects fidelity [11]. Moreover, this model takes into account the effect of higher order modes of the beam that have been ignored by some models. Using this model, the overall crosstalk is estimated including the stray light crosstalk. The SCR of FSOIs system is also evaluated and optimized. The analyses show that the size of the detector and the stray light crosstalk play an important role to optimize the SCR and should be considered when designing a FSOIs system.

\section{OPTICAL FIELD IN A GENERAL MISALIGNED FIRST ORDER OPTICAL SYSTEM}

Figure 1 is a simple schematic diagram for a two-dimensional misaligned optical system. P1 and P2 are two reference planes for the aligned system, and $\mathrm{P} 1 \mathrm{~m}$ and $\mathrm{P} 2 \mathrm{~m}$ are the corresponding planes for the misaligned system. $l$ is the longitudinal length between the input and the output planes. $x_{1}, y_{1}$ and $x_{2}, y_{2}$ are the rectangular coordinates on the misaligned planes $\mathrm{P} 1 \mathrm{~m}$ and $\mathrm{P} 2 \mathrm{~m}$, respectively.

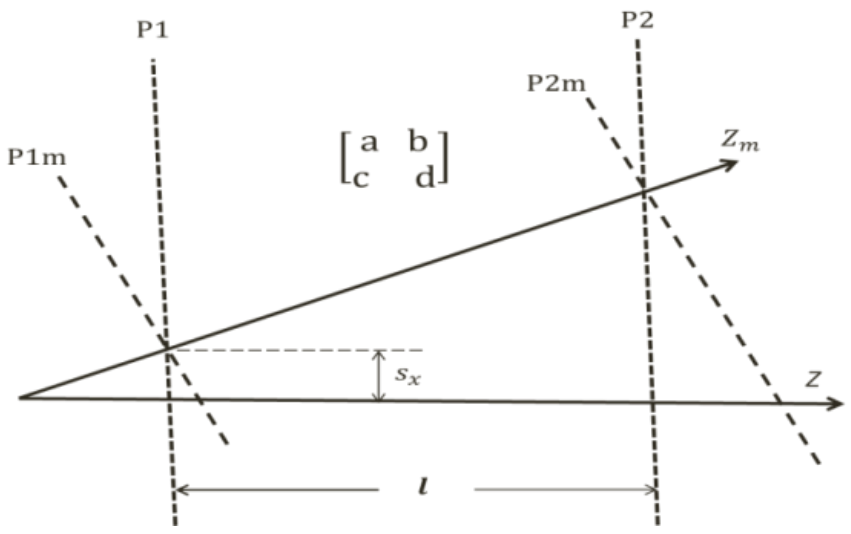

Figure 1. Misaligned optical system

Assuming a linear optical system and using the paraxial approximation, the output optical field $\mathrm{E}_{2}\left(x_{2}, y_{2}\right)$ can be obtained from the input field $\mathrm{E}_{1}\left(x_{2}, y_{2}\right)$ using Collins diffraction integral in rectangular coordinate as [13]: 


$$
\begin{aligned}
& \mathrm{E}_{2}\left(x_{2}, y_{2}\right)=\frac{i k}{2 \pi b} \int_{-\infty-\infty}^{\infty} \int_{1}^{\infty} \mathrm{E}_{1}\left(x_{1}, y_{1}\right) \\
& \times \exp \left[-\frac{i k}{2 b}\left(\begin{array}{l}
a\left(x_{1}^{2}+y_{1}^{2}\right)-2\left(x_{1} x_{2}+y_{1} y_{2}\right) \\
+d\left(x_{2}^{2}+y_{2}^{2}\right)+e x_{1}+f y_{1}+g x_{2}+h y_{2}
\end{array}\right)\right] \mathrm{d} x_{1} d y_{1}
\end{aligned}
$$

$k=2 \pi / \lambda$ is the wave number and $\lambda$ is the wavelength. $\alpha, b, c$, and $d$ are the transfer matrix elements of the aligned optical system and $e, f, g$, and $\mathrm{h}$ are the misalignment parameters and given as :

$$
\begin{aligned}
& e=2\left(\alpha_{T} s_{x}+\beta_{T} \theta_{x}\right) \\
& f=2\left(\alpha_{T} s_{y}+\beta_{T} \theta_{y}\right) \\
& g=2\left(b \gamma_{T}-d \alpha_{T}\right) s_{x}+2\left(b \delta_{T}-d \beta_{T}\right) \theta_{x} \\
& h=2\left(b \gamma_{T}-d \alpha_{T}\right) s_{y}+2\left(b \delta_{T}-d \beta_{T}\right) \theta_{y}
\end{aligned}
$$

$S_{x}$ and $S_{y}$ are the $\mathrm{x}$ and y misalignments $\theta_{x}$ and $\theta_{y}$ are the angular misalignments between the aligned and misaligned transverse axes. $\alpha_{T}, \beta_{T}, \gamma_{T}$, and $\delta_{T}$ are the misalignment elements determined by

$$
\alpha_{T}=1-a, \quad \beta_{T}=l-b, \gamma_{T}=-c, \delta_{T}=1-d
$$

Using $x=\rho \cos (\theta)$ and $y=\rho \sin (\theta),(1)$ in the cylindrical coordinate system can be given by [12]:

$$
\begin{array}{r}
E_{2}\left(\rho_{2}, \theta_{2}\right)=\frac{i k}{2 \pi b} \int_{0}^{\infty} \int_{0}^{2 \pi} E_{1}\left(\rho_{1}, \theta_{1}\right) \exp \left[-\frac{i k}{2 b} \rho_{2}^{\prime 2}\right] \exp \left[\frac{i k a}{2 b} \rho_{1}^{2}\right] \\
\times \exp \left[\frac{i k \rho_{1}}{b} \rho_{2}^{\prime \prime} \cos \left(\theta_{1}-\phi\right)\right] \rho_{1} d \rho_{1} d \theta_{1}
\end{array}
$$

$\rho_{1}, \theta_{1}$ and $\rho_{2}, \theta_{2}$ are the cylindrical coordinates in $\mathrm{P} 1 \mathrm{~m}$ and $\mathrm{P} 2 \mathrm{~m}$ planes, respectively.

$$
\begin{aligned}
& \rho_{2}^{\prime 2}=d \rho_{2}^{2}+g \rho_{2} \cos \theta_{2}+h \rho_{2} \sin \theta_{2} \\
& \rho_{2}^{\prime \prime 2}=\left(\rho_{2} \cos \theta_{2}-e / 2\right)^{2}+\left(\rho_{2} \sin \theta_{2}-f / 2\right)^{2}
\end{aligned}
$$

and

$$
\phi=\tan ^{-1}\left(\frac{\rho_{2} \sin \theta_{2}-f / 2}{\rho_{2} \cos \theta_{2}-e / 2}\right)
$$

The (7) gives the optical field distribution at the output plane for a linear optical system in a cylindrical coordinate system with slight misalignment.

\section{OPTICAL FIELD IN A MISALIGNED FSOIS WITHOUT APERTURE}

Consider the FSOIs system shown in Figure 2. This optical system consists of VCSELs array, microlens array, and detectors array. The lasers array is placed at the focus plane of the transmitter microlens. The misalignments of the microlens with respect to the optical axis of the FSOIs system are $S_{x}, s_{y}=0$ and $\theta_{x}=\theta_{y}=0$ as shown in Figure 2. The transfer matrix elements of the aligned FSOIs system are: 


$$
a=1-\left(l / f_{t}\right), b=l, c=-1 / f_{t}, d=1
$$

and the misalignment parameters are:

$$
\alpha_{T}=l / f_{t}, \beta_{T}=0, \gamma_{T}=1 / f_{t}, \delta_{T}=0
$$

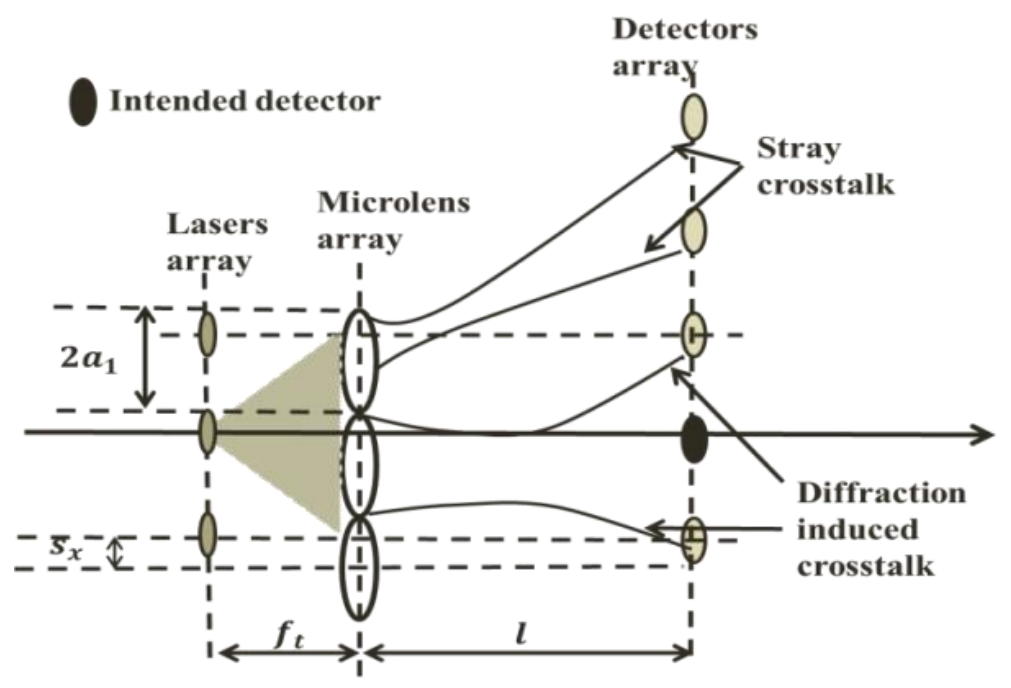

Figure 2. Misaligned lens-based FSOIs

$$
e=2 \frac{l}{f_{t}} s_{x}, f=0, g=0, h=0
$$

The beam emitted from the laser source is a LG beam with waist radius $\omega_{0}$. In this case, the optical field distribution of a LG beam for any order in the cylindrical coordinate system at the plane of the transmitting microlens is given by [5]

$$
\begin{aligned}
\mathrm{E}_{1}\left(\rho_{1}, \theta_{1}\right) & =\left(\sqrt{2} \frac{\rho_{1}}{\omega_{1}}\right)^{m} \exp \left[-\frac{\rho_{1}^{2}}{\omega_{1}^{2}}\right] \\
& \times \mathrm{L}_{\mathrm{p}}^{\mathrm{m}}\left[\frac{2 \rho_{1}^{2}}{\omega_{1}^{2}}\right] \times \exp \left[-i m \theta_{1}\right]
\end{aligned}
$$

$\mathrm{L}_{\mathrm{p}}^{\mathrm{m}}$ is the Laguerre polynomial with mode orders $\mathrm{p}$ and $\mathrm{m}$. Using propagation theory of Gaussian beam in free space, the beam radius at the front surface of the transmitter microlens is

$$
\omega_{1}=\omega_{0} \sqrt{1+\frac{\lambda^{2} f_{t}^{2}}{\pi^{2} \omega_{0}^{4}}}
$$

where $f_{t}$ is the focal length of the transmitter microlens Substituting (14) into (7) and using (11), (12), and (13), we can obtain the optical field at the detectors array as 


$$
\begin{aligned}
& \mathrm{E}_{2}\left(\rho_{2}, \theta_{2}\right)=\left(\frac{i k}{2 l}\right)^{m+1} \exp \left[-i m \theta_{2}\right] \exp \left[\frac{i k}{2 l} \rho_{2}^{2}\right]\left[k^{\prime}+\frac{1}{\omega_{1}^{2}}\right]^{-m-p-1} \\
& {\left[k^{\prime}-\frac{1}{\omega_{1}^{2}}\right]^{p}\left[\frac{\sqrt{2}}{\omega_{1}} \sqrt{\left(\rho_{2} \cos \theta_{2}-2 s_{x} / \mathrm{f}_{\mathrm{t}}\right)^{2}+\left(\rho_{2} \sin \theta_{2}\right)^{2}}\right]^{m}} \\
& \times \exp \left[\frac{-k^{2}\left[\left(\rho_{2} \cos \theta_{2}-2 s_{x} / \mathrm{f}_{\mathrm{t}}\right)^{2}+\left(\rho_{2} \sin \theta_{2}\right)^{2}\right]}{4 l^{2}\left(k^{\prime}+\frac{1}{\omega_{1}^{2}}\right)}\right] \\
& \times L_{p}^{m}\left[\frac{k^{2}\left[\left(\rho_{2} \cos \theta_{2}-2 s_{x} / \mathrm{f}_{\mathrm{t}}\right)^{2}+\left(\rho_{2} \sin \theta_{2}\right)^{2}\right]}{2 \omega_{1}^{2} l^{2}\left(-k^{\prime 2}+\frac{1}{\omega_{1}^{4}}\right)}\right]
\end{aligned}
$$

In the above equation $k^{\prime}$ is

$$
k^{\prime}=\frac{i k\left[1-\left(l / f_{t}\right)\right]}{2 l}
$$

The above equation is closed form formula for the optical field of the LG beam propagating through lens based FSOIs system with slight misalignment. In fact (16) provides a suitable way for estimating the crosstalk in the FSOIs system under consideration, and can be used to study the impact of beam higher order modes on the crosstalk and in turn on the FSOIs system performance.

\section{OPTICAL FIELD IN A MISALIGNED FSOIS WITH CIRCULAR APERTURE USING PROPOSED MODEL}

In this section, a propagation model of an LG beam propagating in a circularly apertured FSOIs system is derived by expressing the hard aperture function of the microlens into in terms of complex Gaussian functions $[14,15]$. Let $A\left(\rho_{1}\right)$ the circular aperture function with $a_{1}$ radius

$$
A\left(\rho_{1}\right)= \begin{cases}1 & \rho_{1} \leq a_{1} \\ 0 & \rho_{1} \succ a_{1}\end{cases}
$$

using (7) and (18), we found the field distribution at the plane of detectors array as:

$$
\begin{aligned}
& \mathrm{E}_{2}\left(\rho_{2}, \theta_{2}\right)=\frac{i k}{2 \pi b} \int_{0}^{\infty} \int_{0}^{2 \pi} \mathrm{E}_{1}\left(\rho_{1}, \theta_{1}\right) A\left(\rho_{1}\right) \\
& \times \exp \left[-\frac{i k}{2 b} \rho_{2}^{\prime 2}\right] \exp \left[\frac{i k a}{2 b} \rho_{1}^{2}\right] \\
& \times \exp \left[\frac{i k \rho_{1}}{b} \rho_{2}^{\prime \prime} \cos \left(\theta_{1}-\varphi\right)\right] \rho_{1} d \rho_{1} d \theta_{1}
\end{aligned}
$$

The above integral can be solved by expressing the circular aperture function in terms of limited number of complex Gaussian functions [14]:

$$
A\left(\rho_{1}\right)=\sum_{n=1}^{N} A_{n} \exp \left(-\frac{B_{n}}{a_{1}^{2}} \rho_{1}^{2}\right)
$$

where $A_{n}$ and $B_{n}$ are complex coefficients. The values of these coefficients for $N=10$ are tabulated in [14]. Substituting (20) into (19) and performing the integration, we obtain 


$$
\begin{aligned}
& \mathrm{E}_{2}\left(\rho_{2}, \theta_{2}\right)=\left(\frac{i k}{2 l}\right)^{m+1} \exp \left[-i m \theta_{2}\right] \exp \left[\frac{i k}{2 l} \rho_{2}^{2}\right] \\
& \times\left[\frac{\sqrt{2}}{\omega_{1}} \sqrt{\left(\rho_{2} \cos \theta_{2}-2 s_{x} / \mathrm{f}_{\mathrm{t}}\right)^{2}+\left(\rho_{2} \sin \theta_{2}\right)^{2}}\right]^{m} \\
& \sum_{n=1}^{N} A_{n}\left[k^{\prime}+\frac{B_{n}}{a_{1}^{2}}+\frac{1}{\omega_{1}^{2}}\right]^{-m-p-1}\left[k^{\prime}+\frac{B_{n}}{a_{1}^{2}}-\frac{1}{\omega_{1}^{2}}\right]^{p} \\
& \times \exp \left[-\frac{k^{2}\left[\left(\rho_{2} \cos \theta_{2}-2 s_{x} / \mathrm{f}_{\mathrm{t}}\right)^{2}+\left(\rho_{2} \sin \theta_{2}\right)^{2}\right]}{4 l^{2}\left(k^{\prime}+\frac{B_{n}}{a_{1}^{2}}+\frac{1}{\omega_{1}^{2}}\right)}\right] \\
& \times L_{p}^{m}\left[\frac{k^{2}\left[\left(\rho_{2} \cos \theta_{2}-2 s_{x} / \mathrm{f}_{\mathrm{t}}\right)^{2}+\left(\rho_{2} \sin \theta_{2}\right)^{2}\right]}{\frac{2 l^{2}}{\omega_{1}^{2}}-2 \omega_{1}^{2} l^{2}\left(k^{\prime}+\frac{B_{n}}{a_{1}^{2}}\right)^{2}}\right]
\end{aligned}
$$

The (21) is the major contribution in this paper and represents an approximate analytical relation for the optical field of the LG beam propagating through the circularly apertured misaligned FSOIs system shown in Figure 2. Note that for infinite aperture size, (21) becomes identical to (16) derived in previous section. In fact, this relation provides a suitable way for estimating the crosstalk in a slightly misaligned FSOIs system taking into account the effect of finite aperture of the microlens.

\section{ESTIMATING CROSSTALK-TO-SIGNAL RATIO}

For the sake of estimating the SCR we consider Figure 2. At the transmitter microlenses array, the overlap between the intended microlens aperture and stray light from neighboring lasers causes a crosstalk noise. The stray crosstalk can be seen as the portion of the transmitted power from the intended source that passes through neighboring microlenses and reach other detectors as shown in Figure 2. The stray crosstalk power can be found as the total power received by all neighboring detectors from the light coming through neighboring microlenses assuming only the intended source is on. In this case, we can estimate the stray crosstalk power by

$$
\begin{aligned}
P_{c 1}=P_{c 11}+P_{c 12} & =4 \iint_{\Omega_{1}}\left|\mathrm{E}_{1}\left(\rho_{1}, \theta_{1}\right)\right|^{2} \rho_{1} d \rho_{1} d \theta_{1} \\
& +4 \iint_{\Omega_{2}}\left|\mathrm{E}_{1}\left(\rho_{1}, \theta_{1}\right)\right|^{2} \rho_{1} d \rho_{1} d \theta_{1}
\end{aligned}
$$

$P_{c 11}$ and $P_{c 12}$ are the crosstalk noises received by the intended detector from the neighbor and next neighbor lasers respectively. $\Omega_{1}$ and $\Omega_{2}$ are the areas covered by neighbor and next neighbor transmitted microlenes, respectively. On the other hand, at the detectors plane, the overlap between the intended detector aperture and the diffracted light beams from other VCSELs through other transmitted microlenes also causes a crosstalk noise. This crosstalk is called diffraction induced crosstalk. The total diffraction induced crosstalk power received by the intended detector can be found as the power received by all neighboring detectors from the light coming through intended microlens power assuming only the intended source is on. In this case, we can estimate the diffraction induced crosstalk power by

$$
\begin{aligned}
P_{c 2}=P_{c 21}+P_{c 22} & =4 \iint_{\Omega_{3}}\left|\mathrm{E}_{2}\left(\rho_{2}, \theta_{2}\right)\right|^{2} \rho_{2} d \rho_{2} d \theta_{2} \\
& +4 \iint_{\Omega_{4}}\left|\mathrm{E}_{2}\left(\rho_{2}, \theta_{2}\right)\right|^{2} \rho_{2} d \rho_{2} d \theta_{2}
\end{aligned}
$$

$P_{c 21}$ and $P_{c 22}$ are the crosstalk noises received by the detector from the neighbor and next neighbor detectors respectively. $\Omega_{3}$ and $\Omega_{4}$ are the areas covered by neighbor and next neighbor detectors, respectively. 
The total power of the crosstalk noise is the sum of $P_{c 1}$ and $P_{c 2}$. The total signal power is the power received by the intended detector from the light coming from the intended source through the intended microlens. Based on this, we estimate the signal power as:

$$
P_{s}=\iint_{\Omega}\left|\mathrm{E}_{2}\left(\rho_{2}, \theta_{2}\right)\right|^{2} \rho_{2} d \rho_{2} d \theta_{2}
$$

$\Omega$ is the area covered by intended detector. After estimating the signal and the crosstalk for the FSOIs system, the SCR could be determined. In our model, no detector preamplifier is used and the crosstalk noise resulting from stray light at transmitted microlenes, light diffraction from apertures, and misalignment are included. Based on (22), (23), and (24), the SCR ratio can be determined as

$$
S C R=\frac{P_{s}}{P_{c 1}+P_{c 2}}
$$

\section{NUMERICAL RESULTS AND DISCUSSION}

The following parameters are used in the simulations: for the laser source the wavelength is $0.850 \mu \mathrm{m}$ and the waist radius of each output beam is $3 \mu \mathrm{m}$; for the transmitter microlens the focal length and the diameter are $720 \mu \mathrm{m}$ and $250 \mu \mathrm{m}$, respectively. The lasers array is placed in the focus plane of the microlens arrays. The interconnection length of is $2.5 \mathrm{~mm}$ and the interconnections spacing is $250 \mu \mathrm{m}$. The SCR will be used as the performance measure. In the simulations, the effect of finite aperture, misalignment, high order modes, and stray crosstalk on the SCR will be emphasized. The simulations are performed using the generalized Collins diffraction theory [16] assuming a linear FSOIs system. To approximate the aperture function we use the same coefficients used in [14] with $N=10$. Figure 3 shows the approximated aperture function with radius $a_{1}=0.125 \mathrm{~mm}$. It is clear that 10 complex Gaussian functions are sufficient to compose the hard aperture function. Simulation results in the following subsections, 6.1 and 6.3, assume only the fundamental $\mathrm{LG}$ mode, $\mathrm{LG}_{00}$, for the laser source. The impact of VCSELs' higher modes will be considered in the subsection 6.3.

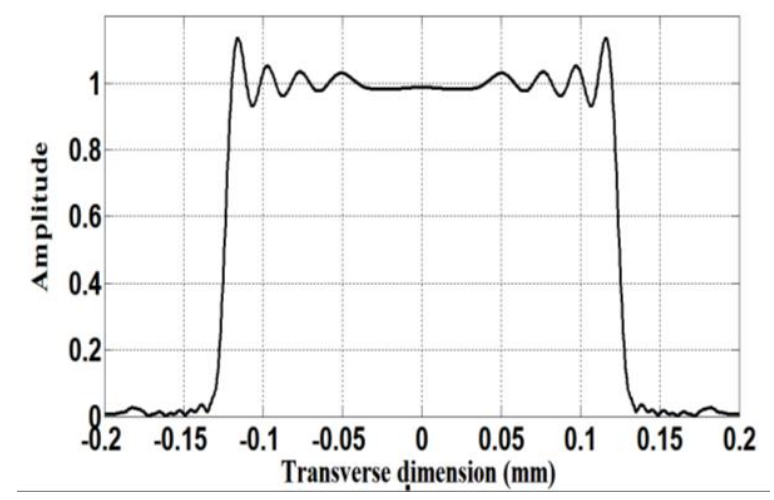

Figure 3. Approximated aperture function with radius $a_{1}=0.125 \mathrm{~mm}$ and $\mathrm{N}=10$

\subsection{SCR for aligned FSOIs system}

Figure 4 shows the SCR versus detector radius for the apertured and the unperturbed FSOIs systems with an interconnection length of $2.5 \mathrm{~mm}$. It is clear that the SCR=is 25 for the apertured FSOIs and it is 23 for the unapertured one. Furthermore, the SCR drops quickly as the detector radius decreases. Moreover, optimum detector radius can be obtained as shown in Figure 4. For example, when the required SCR is above 10 the corresponding detector radius ranges from $5 \mu \mathrm{m}$ to $77 \mu \mathrm{m}$. For maximum SCR, the optimum detector radii for the apertured and the unperturbed systems are $22 \mu \mathrm{m}$ and $23 \mu \mathrm{m}$, respectively. The existence of such optimum can be explained by considering the design formula used to model the crosstalk. In this paper, we consider the diffraction induced cross talk and the stray light crosstalk. The diffraction induced crosstalk 
depends on the detector radius while the stray crosstalk depends on the microlens radius. For a given microlens radius, the stray crosstalk is constant and overwhelms the diffraction crosstalk at small detector size. In this case, the decrease of the SCR as the detector size decreases is due to the decrease in the signal power. For large detector size, the diffraction crosstalk is dominant and increases with the detector size. The balance between the signal power, the diffraction crosstalk, and the stray crosstalk explains the existence of optimum detector size.

To show the impact of changing interconnects density on the design parameters of the FSOIs, we have plotted in Figure 5 the SCR versus detector radius different interconnects spacing. It is noted from the Figure that the SCR decreases as interconnects density increases. For an interconnects spacing of 250 $\mathrm{mm}, 260 \mathrm{~mm}$, and $270 \mathrm{~mm}$ the corresponding optimum values of the SCR are 23, 39, and 67, respectively. Moreover, the corresponding optimum detector radii are $22 \mu \mathrm{m}, 16 \mu \mathrm{m}$, and $13 \mu \mathrm{m}$, respectively. The optimum value of the detector radius decreases as interconnects density decreases. Based on the above simulations and analyses, it appears that the detector size plays an important role and strongly affects the SCR of the FSOIs system.

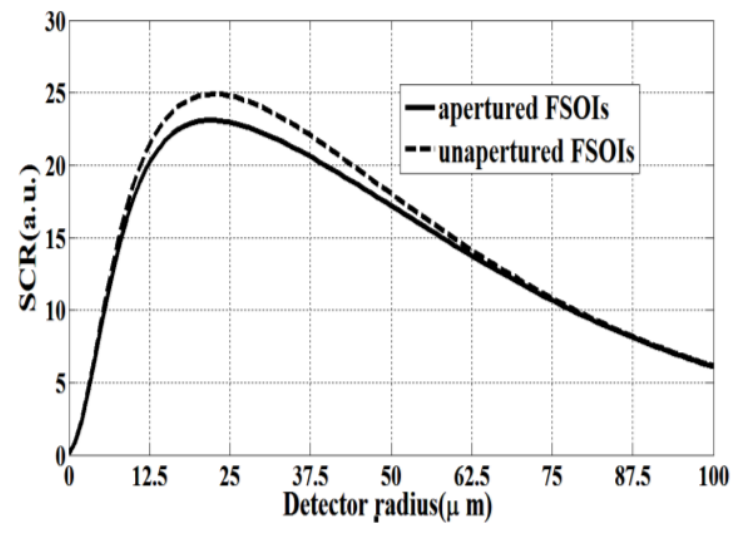

Figure 4. SCR versus detector radius for the apertured and the unperturbed FSOIs system

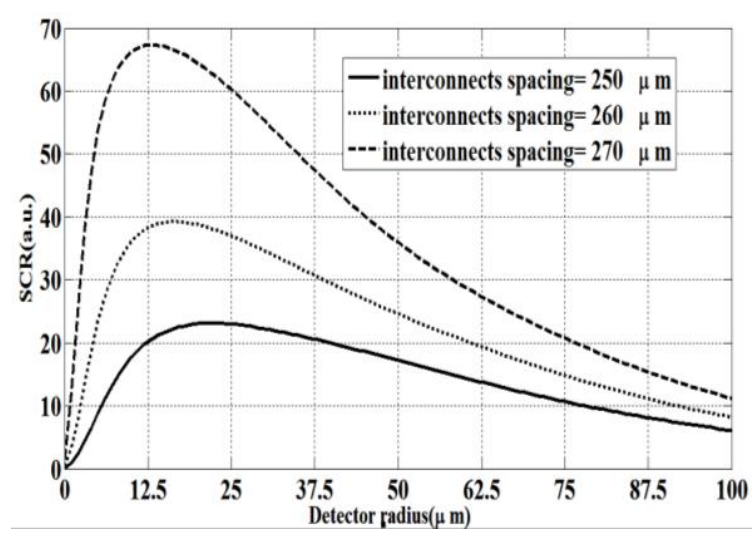

Figure 5. SCR versus detector radius for the apertured FSOIs s with interconnects spacing as a parameter

\subsection{SCR for misaligned FSOIs system}

Figure 6 shows the SCR versus detector radius for the apertured FSOIs system with an interconnection length of $2.5 \mathrm{~mm}$ under different misalignments for the microlens in X-direction. The SCR decreases with misalignment. The SCR is 15.5 for $5 \mu \mathrm{m}$ misalignment, while it decreases to 10.5 when the misalignment is $10 \mu \mathrm{m}$. Moreover, the optimum detector radius decreases with increasing the misalignment. This can be explained by the aid of using Figure 7. It is clear that the misalignment strongly affects the normalized crosstalk power plotted as in Figure 8. Therefore, optimum design will choose small detector size to minimize the crosstalk and in turn improve the SCR.

\subsection{Effect of VCSELs' higher modes on SCR}

In Figure 8, we have plotted the SCR versus detector radius for the apertured FSOIs system in the presence of high order modes for the laser source. We assumed the presence of $L G 00, L_{00}+L_{01}$, $\mathrm{LG}_{00}+\mathrm{LG}_{01}+\mathrm{LG}_{10}$, respectively. The fractional coefficients in the two modes case for $\mathrm{LG}_{00}, \mathrm{LG}_{01}$ are 0.9 and 0.1 , respectively, while they are $0.4,0.55$, and 0.05 for $\mathrm{LG}_{00}, \mathrm{LG}_{01}$, and $\mathrm{LG}_{10}$ respectively, for the three modes [5], [17]. We can see from Figure 8 that the SCR, in the presence of only the $\mathrm{LG}_{00}$ mode, is higher than that when two or three modes are present. For example, the optimum SCR for the $\mathrm{LG}_{00}$ mode is 23 . However, the optimum SCR for the $\mathrm{LG}_{00}+\mathrm{LG}_{01}$ modes and $\mathrm{LG}_{00}+\mathrm{LG}_{01}+\mathrm{LG}_{10}$ modes have dropped to approximately 14 and 2.7, respectively. The corresponding optimum detector radii for single mode, two modes, and three modes are $22 \mu \mathrm{m}, 28 \mu \mathrm{m}$, and $42 \mu \mathrm{m}$, respectively. The optimum detector radius increases with number of modes. This can be explained based on the fact that the spread of the light increases with number of modes and the detector radius should increase to gather more light for larger SCR. 


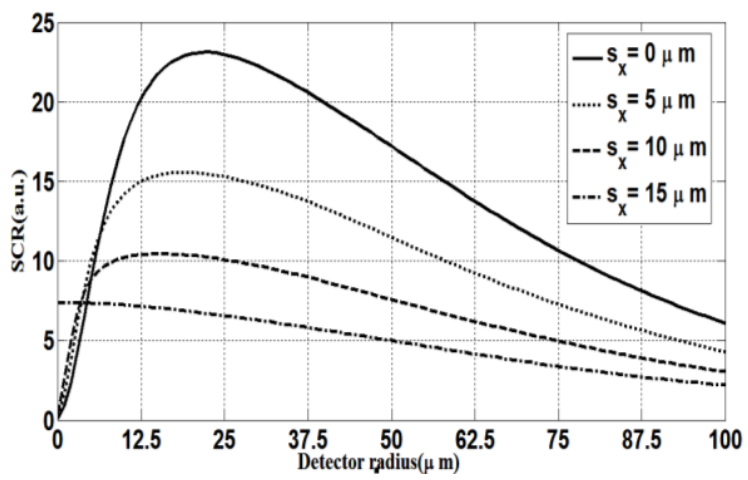

Figure 6. SCR versus detector radius for the apertured FSOIs system with $\mathrm{x}$-misalignment as a parameter

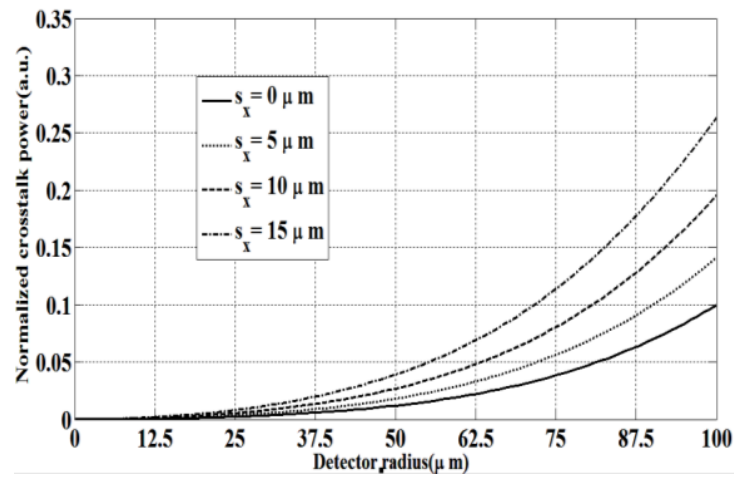

Figure 7. Normalized crosstalk versus detector radius for the misaligned apertured FSOIs

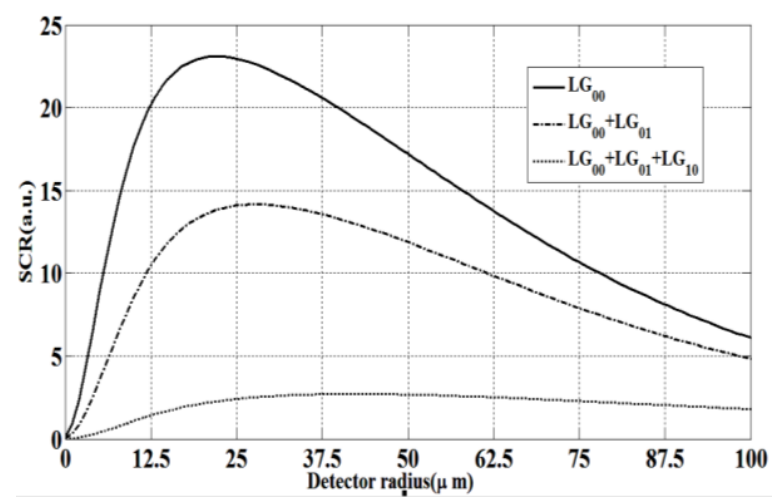

Figure 8. SCR versus detector radius for the apertured FSOIs system with different laser modes

\section{CONCLUSION}

We have analyzed a slightly misaligned free space optical interconnects system employing arrays of laser sources, microlenses, and detectors. A novel diffraction model has been proposed using cylindrical form of Collins diffraction integral. Some approximate closed form formulas for the light optical field at the detector plane were derived. Using the proposed diffraction model and the derived formulas, the SCR has been estimated and used as the performance measure. Numerical simulations have shown that misalignment, stray light, microlens finite aperture, and laser higher order modes affect the free space optical interconnects system and have strong influences on the signal-to-crosstalk ratio, and it is important to consider them in real applications. Moreover, our analyses have shown that optimum SCR can be obtained using the detector size as optimization parameter.

\section{REFERENCES}

[1] Tang, Suning; Chen, Ray T.; Garrett, Lara; Gerold, Dave; Li, Maggie M. ”Design limitations of highly parallel free-space optical interconnects based on array of vertical cavity surface-emitting laser diodes, microlenses, and photo detectors," J. Lightwave Technol. 12, 1971-1975, 1994.

[2] Fuada, Syifaul, et al. "Noise Analysis in VLC Optical Link based Discrette OP-AMP Trans-Impedance Amplifier (TIA)," Telkomnika 15.3, 1012-1021, 2017.

[3] Al-ababneh, Nedal. "Aberrated Microlenses to Reduce Crosstalk in Free Space Optical Interconnects Systems," Modern Applied Science 12, no. 5, 100, 2018.

[4] M. Châteauneuf, A. G. Kirk, D. V. Plant, T. Yamamoto, and J. D. Ahearn, "512-channel vertical-cavity surfaceemitting laser-based free-space optical link," Appl. Opt. 41, 5552-5561, 2002.

[5] F. F. Tsai, C. J. O'Brien, N. S. Petrovic', and A. D. Rakic',"Analysis of optical channel cross talk for free-space optical interconnects in the presence of higher-order transverse modes," Appl. Opt. 44, 6380-6387, 2005.

[6] R. Wong, A. D. Rakic, and M. L. Majewski, "Design of microchannel free-space optical interconnects based on vertical-cavity surface-emitting laser arrays," Appl. Opt., vol. 41, no. 17, 3469-3478, 2002. 
[7] N. S. Petrovic' and A. D. Rakic', "Modeling diffraction and imaging of laser beams by the mode-expansion method," J. Opt. Soc. Am. B 22, 556-566, 2005.

[8] N. S. F. Ozkan, W. L. Hendrick, P. J. Marchand, and S. C. Esener, "Misalignment tolerance analysis of free-space optical interconnects via statistical methods," Appl. Opt. 41, 2686-2694, 2002.

[9] F. Lacroix, M. Châteauneuf, X. Xue, and A. G. Kirk, "Experimental and numerical analyses of misalignment tolerances in free-space optical interconnects," Appl. Opt. 39, 704-713, 2000.

[10] Wenhua Hu, Xiujian Li, Jiankun Yang, and Di Kong "Crosstalk analysis of aligned and misaligned free-space optical interconnect systems," J. Opt. Soc. Am. A 27, 200-205, 2010.

[11] N. Davidson, A. A. Friesem, and E. Hasman,"On the limits of optical interconnects," Applied Optics 31, 5426-5430, 1992.

[12] Y. Cai S. He, "Propagation of a Laguerre-Gaussian beam through a slightly misaligned paraxial optical system" Appl. Phys. B 84, 493-500, 2006.

[13] S. Wang, L. Ronchi, "Principles and design of optical arrays, in Progress in Optics 25," ed. by E. Wolf (NorthHolland, Amsterdam, 1988.

[14] J. J. Wen and M. A. Breazeale, "A diffraction beam field expressed as the superposition of Gaussian beams," J. Acoust. Soc. Am. 83, 1752-1756, 1988.

[15] D. Ding and Y. Zhang, "Notes on the Gaussian beam expansion," J. Acoust. Soc. Am. 116, 1401-1405, 2004.

[16] S. A. Collins, "Lens-systems diffraction integral written in terms of matrix optics," J. Opt. Soc. Am. 60, 1168-1177, 1970.

[17] Rong Wang, Aleksandar D. Rakić, Marian L. Majewski, "Analysis of lensless free-space optical interconnects based on multi-transverse mode vertical-cavity-surface-emitting lasers," Optics Communications 167, 261-271, 1999.

\section{BIOGRAPHY OF AUTHOR}

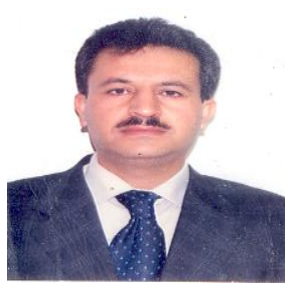

Prof. Nedal Al-Ababneh received his B.Sc. and M.Sc. degrees in Electrical Engineering from Jordan University of Science and Technology in 1993 and 1996 respectively. He received his Doctoral of Engineering degree in Electrical engineering from University of MassachusettsLowell (USA) in 2004. He is currently full professor with the department of electrical engineering in Jordan University of Science and Technology. His research interests are in free space optical interconnects and distributed detection systems. 